Bolm Inst. oceanogr., S Paulo, 31(1):39-53, 1982

\title{
ALGUNS ASPECTOS METODOLÓgICOS DA MEDIDA DA EXCREÇÃO DE MATÉRIA ORGÂ- NICA PELO FITOPLÂNCTON POR ESPECTROMETRIA DE CINTILAÇÃO LİQUIDA*
}

\author{
Armando A. H. VIEIRA ${ }^{1}$ \& Elizabeth AIDAR-ARAGÃO ${ }^{2}$ \\ 1 Departamento de Ciências Biológicas, Universidade Federal de São Carlos \\ 2 Instituto Oceanográfico da Universidade de São Paulo
}

\section{Synopsis}

Some aspects of the procedures to measure dissolved organic matter (DOM) released by phytoplankton account for important sources of errors. These aspects include: cell damage upon filtration of samples, residual inorganic ${ }^{1{ }^{4} \mathrm{C}}$ in the filtrates,

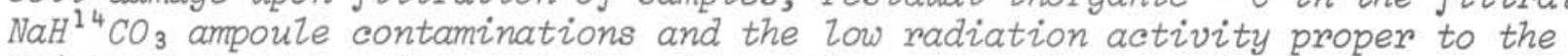
released samples. Some experiments on these procedural problems were carried out and their effects on the released DOM were evaluated. A procedure to use control samples to avoid errors in the technique of DOM analysis by the liquid cintillation counter is described. Experiments on the validity of stocking preserved filtrates of natural plankton populations and algal cultures were also carried out for long periods prior to the radioactivity measurements.

\section{Introdução}

Pode-se situar o início do estudo da excreção de matéria orgânica dissolvida (MOD) pelo fitoplâncton no começo do século, com o trabalho de pütter (1908a, cit. in: Jфrgensen, 1976). Verificando as necessidades nutricionais de vārios invertebrados filtradores marinhos, Pütter admitiu que eles seriam incapazes de processar o grande volume de água necessário para obter o alimento suficiente. Parte do alimento, portanto, deveria vir da MOD. 0 autor, em trabaTho subseqüente (1908b, cit. in:

J $\phi$ rgensen, op. cit.), inferiu, baseando-se em medidas de MOD na água do mar, que somente uma porcentagem muito pequena de carbono assimilado pelo fitoplâncton era incorporada às células e que o restante era liberado para o meio. Contudo, as técnicas usadas pelo autor para a medida de MOD deram resultados errôneos, muito altos (Ehrhardt, 1977), superestimando a excreção do fitoplâncton. Posteriormente, Krogh \& Keys (1934) estabeleceram uma metodologia mais precisa que a utilizada por Pütter. Baseando-se em medidas de MOD nos oceanos, posteriormente, Krogh $(1934 a, b)$ concluiu ser impossível que organismos planctônicos, durante o seu crescimento, eliminassem matéria orgânica para o meio. Essa conclusão, tão bem fundamentada, e traba-

* Trabalho realizado no Instituto Oceanográfico da U.S.P. com suporte financeiro da FINEP.

Publ. n! 530 do Inst. oceanogr. da Usp.
Thos como os de Krogh, Lange \& Smith (1930) e Myers \& Johnston (1949) - que mostraram que, em culturas de cloroficeas, a liberação de produtos extracelulares não era maior que $5 \%$ do carbono total assimilado - fizeram com que os pesquisadores não se interessassem pela excreção do fitoplâncton até o final da década de 50. Nessa época, trabalhos como os de Braarud \& Foyn (1930, cit. in: Fogg, 1958), que mostraram liberação de até $30 \%$ do carbono assimilado em culturas de Chlamydomonas sp, passaram desapercebidos.

Com a introdução da metodologia dos traçadores radioativos, fazendo-se uso do ${ }^{14} \mathrm{C}$ nas medidas de produção primária (Steemann-Nielsen, 1952), e com o aperfeiçoamento das técnicas de análise da água do mar na década de 60 , foram reiniciados os estudos da excreção do fitoplâncton.

Fogg (1958) adaptou esse método do ${ }^{14} \mathrm{C}$ ao estudo da excreção com medidas feitas em detectores Geiger-Müller. A introdução da técnica de detectores de radiação com soluções cintiladoras (1îquido cintilador) por Anderson \& Zeutschel (1970) permitiu alcançar sensibilidade maior na determinação quantitativa da MOD excretada pelo fitoplâncton.

Para a quantificação da excreção por esse método, existem duas técnicas bāsicas: a) fotooxidação por luz ultravioleta da MOD excretada, com posterior captação do ${ }^{14} \mathrm{CO}_{2}$ por hiamina em líquido cintilador (Armstrong et al., 1966; 
Bolm Inst. oceanogr., S Paulo, 31(1), 1982

Thomas, 1971; Berman \& Holm-Hansen, 1974); b) medidas diretas da radioatividade de alíquotas dos filtrados adicionadas ao líquido cintilador

(Anderson \& Zeutschel, op. cit.). Na primeira tëcnica, a MOD marcada existente no volume total dos filtrados é transformada em ${ }^{14} \mathrm{CO}_{2}$ por fotooxidação, incorporada pela hiamina, adicionada ao líquido cintilador e, depois, medida a sua radioatividade. Na segunda técnica, são utilizadas apenas alíquotas dos filtrados. Este detalhe faz aumentar a precisão da primeira, em relação à segunda. No entanto, a fotooxidação da MOD e a captação do ${ }^{14} \mathrm{CO}_{2}$, necessārias no primeiro caso, requerem condições especiais que tornam a técnica de difícil utilização em trabalhos no "campo" ou quando um grande número de amostras deva ser analisado. Uma vantagem da segunda técnica é que ela envolve, apenas, a acidificação e o borbulhamento dos filtrados, na preparação das amostras para as medidas de radioatividade. Outra vantagem, é que as amostras podem ser contadas quase que imediatamente após terminada a incubação.

Entretanto, uma questão tem sido levantada por värios autores (Arthur \& Rigler, 1967; Steemann-Nielsen, 1975; Williams \& Yentsch, 1976; Bul'Yon, 1976; Sellner etal.,1976). A excreção de MOD é realmente um processo natural e normal em células saudâveis do fitoplâncton? Ou a radioatividade observada nos filtrados seria apenas um produto das técnicas metodológicas? Esses autores (op. cit.) põem em dúvida as altas taxas de excreção encontradas por diversos pesquisadores e apresentam a hipótese de que isso se deva, principalmente, à metodologia rotineiramente empregada e a choques causados por variações ambientais durante o processamento laboratorial das amostras.

Nas duas técnicas de quantificação, os autores (op. cit.) apontam como importantes fontes de erro, principalmente, a ruptura de células durante a filtração, $0{ }^{14} \mathrm{C}$ inorgânico não eliminado completamente pelo processo de borbu1hamento e as contaminações das ampolas de $\mathrm{NaH}^{14} \mathrm{CO}_{3}$ por outros compostos radioativos orgânicos, que permaneceriam nos filtrados após o borbulhamento. Estes problemas, aliados à baixa concentração radioativa das amostras de excreção, superestimariam a excreção.
Estes aspectos são avaliados neste trabalho, procurando-se, através de um refinamento da técnica utilizada, minimizar os erros inerentes às medidas da MOD proveniente da excreção do fitoplâncton.

\section{Metodologia básica}

$\mathrm{Na}$ maior parte dos testes realizados, foi usada água do mar filtrada, adicionando-se traços de $\mathrm{NaH}^{14} \mathrm{CO}_{3}$ e/ou glicerol- ${ }^{14} \mathrm{C}$. Em outros, foram empregadas culturas unialgais de Phaeodactylum tricornutum, Skeletonema costatum e Tetraselmis sp, mantidas em meio Guillard-f (Guillard \& Ryther, 1962).

Para a filtração das amostras (após a incubação em presença de $\mathrm{NaH}^{14} \mathrm{CO}_{3}$, no caso das culturas de algas), preparou-se um conjunto de filtradores para processar seis amostras, simultaneamente. Este conjunto consta de seis filtradores Millipore-Xx1004700, 1igados em série, por um tubo metálico (Fig. 1). Foram utilizados filtros de membrana Millipore HA de $47 \mathrm{~mm}$ de diâmetro, para facilitar a filtração das amostras sob baixa pressão de vácuo e reduzir a eventual ruptura das células. A pressão empregada foi de $1 / 3$ de atm.

$0 \mathrm{pH}$ dos filtrados foi, em geral, ajustado para 2,8 a 3,0, com HC1 - $10 \mathrm{~N}$, salvo no caso em que a finalidade do experimento era verificar o efeito do $\mathrm{pH}$. Após as filtrações, adaptou-se, às mangueiras que ligavam os kitasatos ao tubo metálico, ponteiras de vidro (pipetas tipo Pasteur), que foram imersas nos filtrados, para borbulhamento (Fig. 2). Este foi feito por um período de $30 \mathrm{mi}-$ nutos, com ar filtrado em algodão. Para a captação do ${ }^{14} \mathrm{CO}_{2}$ formado, 1 igou-se os kitasatos a um frasco-lavador com solução de $\mathrm{NaOH}-10 \mathrm{~N}$. Terminado o borbulhamento, o pH dos filtrados pode ser reajustado para o valor original da amostra, mas testes preliminares mostraram ser desnecessário esse procedimento.

Alíquotas de $10 \mathrm{ml}$ dos filtrados foram retiradas em duplicata e transferidas para frascos de cintilação, aos quais foram adicionados $10 \mathrm{~m} 1$ do líquido cintilador Instage1 (Packard).

A atividade da matéria orgânica particulada e da dissolvida (excretada) foi medida em espectrômetro de radiação $\beta$ (Sistema detector com solução cintiladora) Packard, mod. Tri Carb C-2425, mantido numa sala refrigerada a $20^{\circ} \mathrm{C}$. 
A eficiência de contagem, calculada pelo método do padrão externo do aparetho (Wang \& Willis, 1965), variou de 78 a $83 \%$ nas medidas dos excretados e de 85 a $90 \%$ naquelas da matéria orgânica particulada. As amostras dos filtrados foram contadas durante 100 minutos, ou no mínimo 10.000 impulsos, para assegurar um erro padrão menor que $2 \%$, ao nível de $p=0,05$. Os filtros foram contados até 40.000 impulsos, com um erro padrão de $0,7 \%$.

As ampolas de $\mathrm{NaH}^{14} \mathrm{CO}_{3}$ foram padronizadas da seguinte maneira: uma ampola foi diluỉda em $50 \mathrm{ml}$ de solução Bray (Bray, 1960) com Hyamina 10x (Packard) a $10 \%$. Dez subamostras de $1,0 \mathrm{ml}$ dessa solução foram transferidas para frascos de cintilação, contendo $9,0 \mathrm{ml}$ de solução Bray. Foram preparadas 10 réplicas para cada ampola e contadas 4 vezes cada uma. A eficiência de contagem foi de $90 \%$.

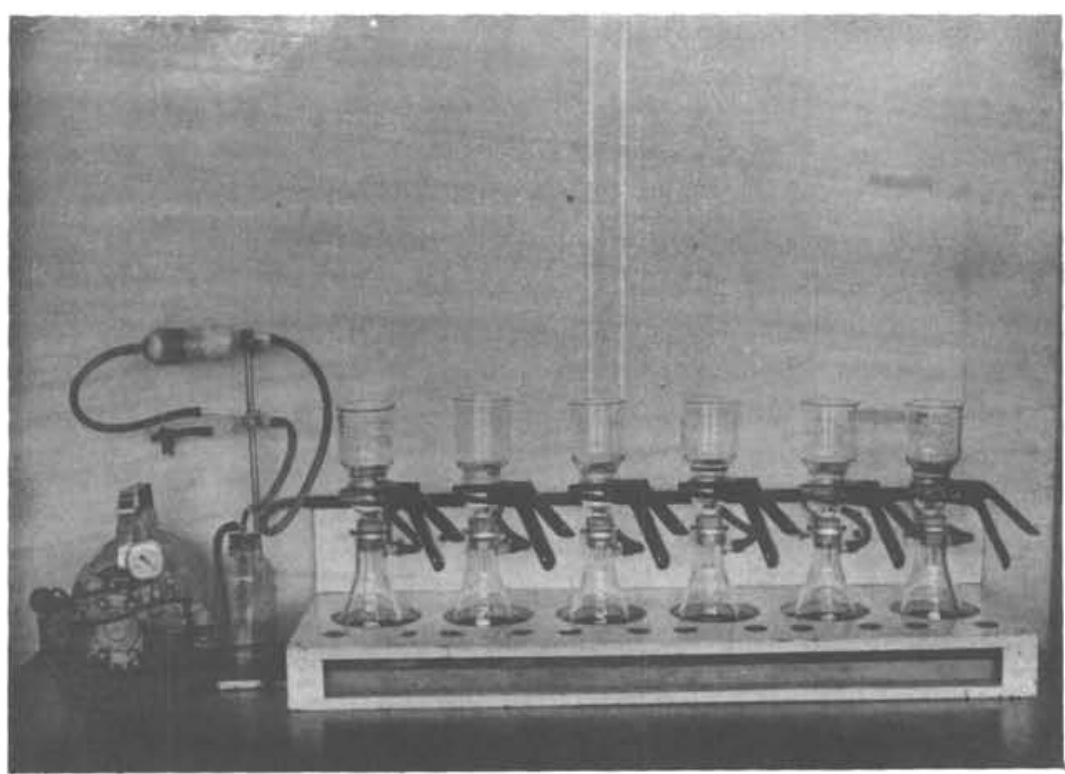

Fig. 1. Conjunto para filtração simultânea das amostras.

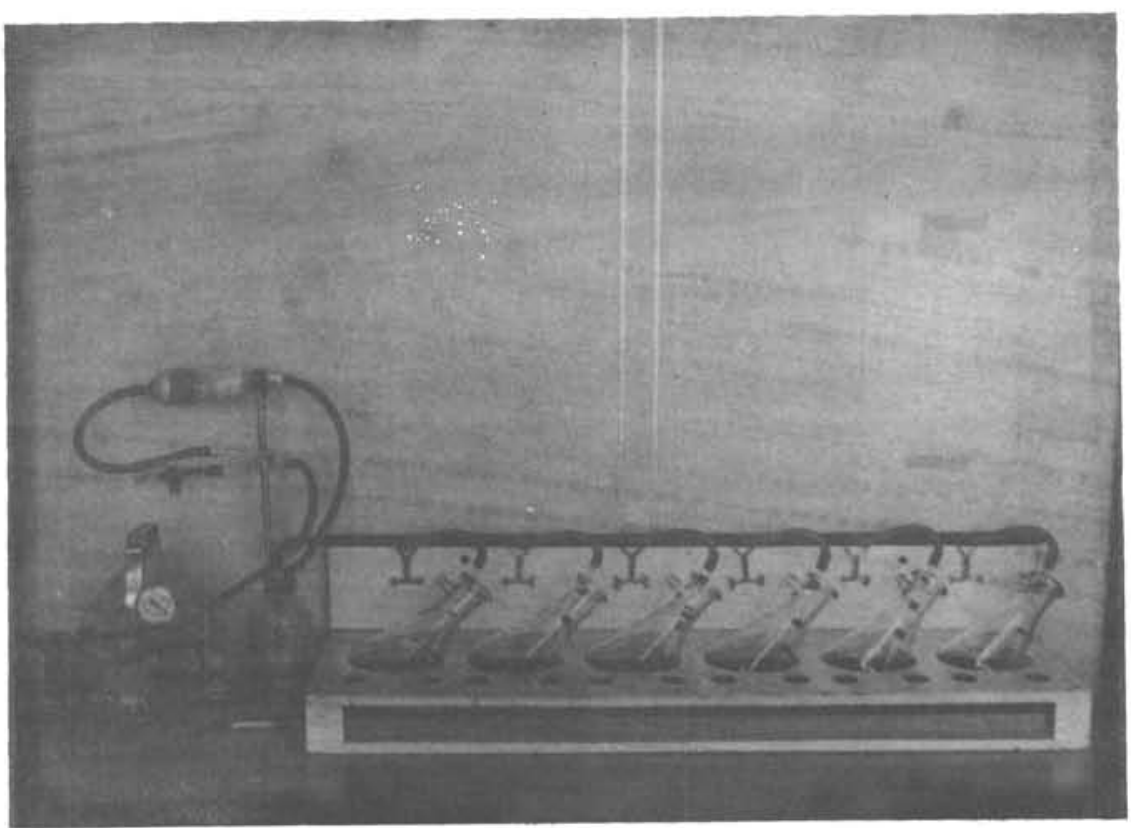

Fig. 2. Conjunto de filtração mostrando a posição dos kitasatos durante o borbulhamento dos filtrados. 
Bolm Inst. oceanogr., S Paulo, 31(1), 1982

Resultados e discussão

os experimentos realizados são, a seguir, apresentados e seus resultados discutidos.

\section{Eliminacão do ${ }^{14} \mathrm{C}$-inorgânico dos filtrados}

Para se testar o tempo de borbulhamento e o $\mathrm{pH}$ ótimo para a eliminação completa do ${ }^{14} \mathrm{C}$ inorganico dos filtrados, foi feito um experimento semelhante ao de Smith Jr. (1975).

A solução de $\mathrm{NaH}^{14} \mathrm{CO}_{3}$ foi adicionada a $1100 \mathrm{ml}$ de água do mar filtrada. Foram retiradas, desta, cinco subamostras de $200 \mathrm{ml}$, que tiveram o seu $\mathrm{pH}$ ajustado com HC1- $10 \mathrm{~N}$ para 2,0, 2,5, $3,0,3,5$ e 4,0, respectivamente. Em seguida, cada uma delas foi borbulhada durante $5,10,15,30,60$ e 180 minutos. Ao final destes tempos, foram retiradas alíquotas para contagem do ${ }^{14} \mathrm{C}$-inorgânico residual, por cintilação

Na Tabela I, são apresentados os resultados das cinco subamostras em número de desintegrações por minuto (DPM), em relação ao tempo de borbulhamento. Como se vê, com 5 minutos de borbulhamento e valor de $\mathrm{pH}$ igual ou superior a 3,5 , a eliminação do ${ }^{14} \mathrm{C}$ inorgânico não è total. Smith Jr. (op. cit.) encontrou um tempo ótimo de 5 - 10 minutos e um $\mathrm{pH}$ ótimo de 3,0 . Para garantir a completa eliminação do ${ }^{1{ }^{4} \mathrm{C}}$ inorgânico, sugere-se o uso do tempo de borbulhamento de 30 minutos e abaixamento do $\mathrm{pH}$ dos filtrados para $2,8-3,0$.

Chama-se a atenção para a atividade relativamente alta das amostras, quando comparadas com o $\mathrm{Bg}$ (amostra controle com água do mar sem $\mathrm{NaH}^{14} \mathrm{CO}_{3}$ ), mesmo depois de 180 minutos de borbulhamento. Este problema será abordado adiante.

\section{Solucão cintiladora}

A solução cintiladora ideal é a que acusa o maior índice de mérito (M), definido como $\mathrm{M}=\mathrm{E} \cdot \mathrm{V}$, onde

E é a eficiência de contagem e

$\mathrm{V}$ é a porcentagem da amostra aquosa incorporada pela solução cintiladora (Brandsome, 1970).

Ainda a solução cintiladora, mais a amostra, devem formar emulsão homogênea em temperatura exequível de trabalho, isto é, possível de se obter em sala com um condicionador de ar comum, se o medi- dor de radioatividade não dispuser de sistema refrigerador. A estabilidade da emulsão água - líquido cintilador está relacionada com a temperatura e dela depende, também, a eficiência das contagens. Por exemplo, a solução ToluenoPPO-dimetil POPOP-BioSolv necessita de uma temperatura de $8,5^{\circ} \mathrm{C}$ para incorporar $2 \mathrm{~m} 1$ de água salgada (Anderson \& Zeutschel, 1970).

Atualmente, existem disponíveis, comercialmente, soluções cintiladoras jā preparadas, com grande poder de incorporação de amostras aquosas, como Aquasol (Beckman) e Instagel (Packard). Soluções com relativo poder de incorporação e de custo menor, podem ser preparadas com Triton- $\mathrm{x}-100$ (Patterson \& Greene, 1965; Turner, 1968). No presente trabalho, foi utilizada a solução Instagel.

\section{Tabela I - ${ }^{14} \mathrm{C}$-inorgânico residual (em DPM) em relação ao tempo de borbulhamento e ao $\mathrm{pH}$ dos filtrados; mëdias de 2 rē- plicas e 4 contagens cada}

\begin{tabular}{cccccc}
\hline $\begin{array}{c}\text { Tempo de } \\
\text { borbulhamer to } \\
\text { (minutos) }\end{array}$ & 2,0 & 2.5 & 3.0 & 3.5 & 4,0 \\
\hline 5 & 134 & 1 & 134 & 144 & 162 \\
10 & 136 & 136 & 132 & 133 & 135 \\
15 & 131 & 131 & 133 & 131 & 136 \\
30 & 131 & 132 & 130 & 135 & 138 \\
60 & 130 & 128 & 131 & 134 & 136 \\
180 & 128 & 128 & 131 & 133 & 133 \\
\hline
\end{tabular}

Bg (somente ägua do mar) $=65 \mathrm{OPM}$

Atividade adicionada $=47.087 .950 \mathrm{DPM} / 200 \mathrm{ml}$

\section{Volume da alíquota dos filtrados}

Devido à baixa atividade que a MOD excretada geralmente apresenta, é conveniente que o volume utilizado dos filtrados seja o maior possíve1. Porém, estes devem ser incorporados pelo Instage1, formando uma "mistura homogênea" e estáve1.

De acordo com Turner (op. cit.), preparou-se uma série de frascos de cintilação com $10 \mathrm{ml}$ de Instage:l e uma quantidade fixa $(1,0 \mathrm{ml})$ de solução Glicero $1-{ }^{14} \mathrm{C}$ em água do mar filtrada e esterilizada. A atividade desta solução era de $0,0006 \mu \mathrm{Ci} / \mathrm{ml}$. A variação dos volumes na série de frascos, de 1,0 a $10,0 \mathrm{ml}$, foi feita com água do mar filtrada. 
As contagens (em número de desintegrações por minuto-DPM) variaram com o volume adicionado de água do mar. Essa variação ocorreu entre 2,5 e $5,0 \mathrm{ml}$ (Fig. 3) $\dot{\sim}$ Nessa faixa, não ocorre incorporação completa da água pelo Instagel, havendo formação de duas fases.

Para os demais volumes, considerando-se - erro de $0,7 \%$ de contagem das amostras, repetiu-se o valor de 1332 DPM, correspondente à ativiaade adicionada de $0,0006 \mu \mathrm{Ci}$. A partir de $6 \mathrm{ml}$, a incorporação da água pelo Instagel é completa. A atividade das amostras com $10 \mathrm{ml}$ de água foi, praticamente, a mesma obtida nas amostras com $1 \mathrm{ml}$ de àgua. Nestas, a eficiência de detecção foi de $90 \%$.

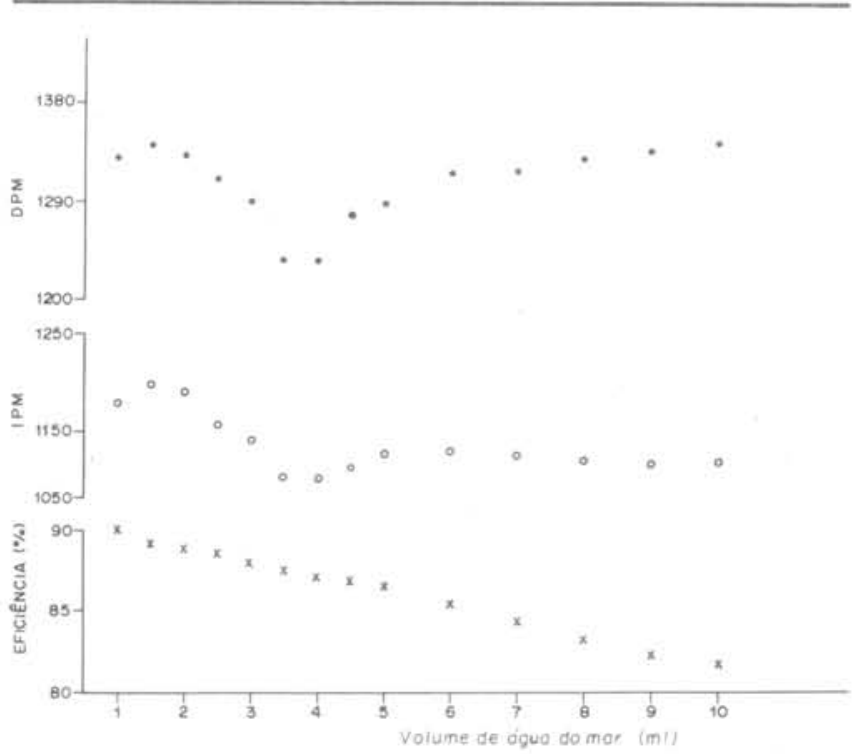

Fig. 3. Variação da eficiência de contagem $(x)$, do número de impulsos por minuto, IPM (o), e do nümero de desintegrações por minuto, $\operatorname{DPM}(\bullet)$, com o volume da alíquota da ägua do mar adicionada. Atividade adicionada (Glicerol $-{ }^{14} \mathrm{C}=1332$ DPM).

\section{Efeito da temperatura}

A emulsão de Instagel com água do mar na proporção de $1: 1(\mathrm{v}: \mathrm{v})$ apresentou-se diferente en várias temperaturas testadas. Entre 2 e $12^{\circ} \mathrm{C}$, ela é translúcida, em forma de gel consistente, mantendo-se com ünica fase por até 60 dias; entre 12 e $22^{\circ} \mathrm{C}$, seu aspecto é opalescente e o gel é mais fluido que nas temperaturas anteriores, mantendo-se estável, sem separação de fases por várias semanas; de
22 a $30^{\circ} \mathrm{C}$, torna-se de coloração leitosa, mais fluida, e a separação de fases ocorre rapidamente.

\section{Reprodutibilidade das réplicas}

Para se verificar a reprodutibilidade das réplicas, considerando a variação de volume da MOD marcada, efetuou-se - seguinte teste: preparou-se uma série de frascos de cintilação com $10 \mathrm{ml}$ de Instagel e volumes variáveis ( 1 a $10 \mathrm{ml}$ ) de uma solução de glicerol- ${ }^{14} \mathrm{C}$, em ägua do $\operatorname{mar}(464 \mathrm{DPM} / \mathrm{ml})$. Para cada volume, foram preparadas cinco réplicas. Na Figura 4, são apresentados os coeficientes de variação das cinco réplicas (média de duas contagens para cada réplica) e os coeficientes de variação de seis contagens de uma só réplica de cada volume de glicerol- ${ }^{14} \mathrm{C}$ (MOD marcada), escolhida ao acaso. Como se pode verificar, os valores dos coeficientes são maiores na faixa onde ocorre a formação de duas fases.

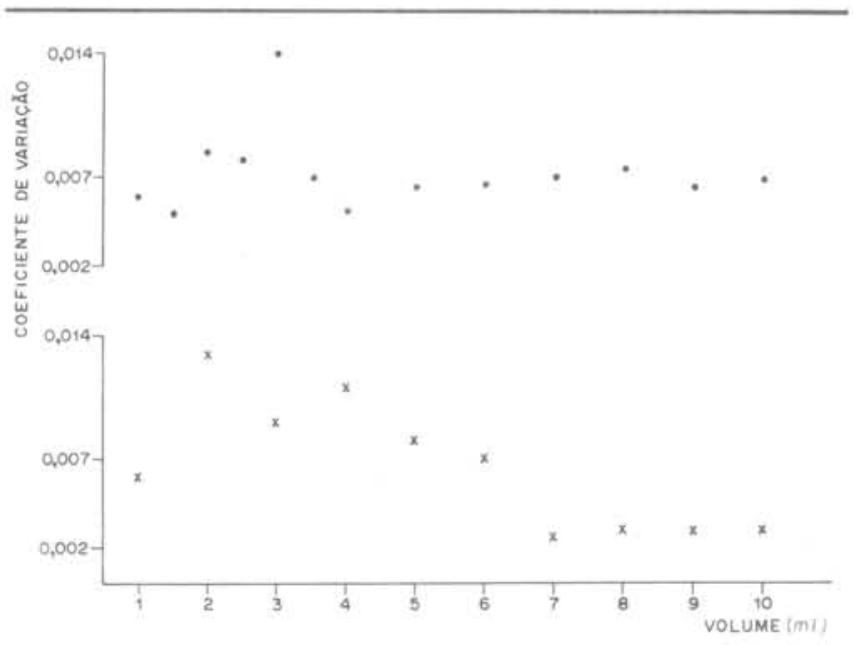

Fig. 4. Coeficientes de variação das contagens de cinco réplicas de cada volume $(x)$ e de seis contagens de uma sö rēplica de cada volume

(•) da amostra de ägua do mar com glicerol $-1{ }^{14} \mathrm{C}$.

\section{Efeito do volume na exatidão das medidas}

Outro problema referente à solução cintiladora, diz respeito à exatidà das medidas; is to é, se contagens de vólumes diferentes da mesma amostra radioativa reproduzem sempre a atividade dessa amostra. Essa é uma questão de extrema importância, já que o Carbono-14 é um emissor puro de partículas $\beta$ de baixa energia. Estas podem ser bloqueadas 
pelas micropartículas de āgua salgada em emulsão e, portanto, não ser eficientemente captadas (Brandsome, 1970). Is to acarretaria uma sub-estimativa da MOD total. Para se averiguar este aspecto, foi preparada uma série de frascos de cintilação com $10 \mathrm{ml}$ de Instage 1 e quantidades crescentes de uma solução de glicerol- $-{ }^{14} \mathrm{C}$ em àgua do mar $(0,025$ $\mu \mathrm{Ci} / \mathrm{ml})$. O volume de água salgada, em cada frasco, foi completado para $10 \mathrm{ml}$, adicionando-se água filtrada. Para cada concentração de glicerol ${ }^{14} \mathrm{C}$, foram feitas duas réplicas.

Pela Tabela II, observa-se uma relação linear entre as atividades (em DPM) e o volume utilizado da solução marcada. A variação média das contagens, considerando-se os resultados em DPM/ml, foi de $0,6 \%$; dentro, portanto, do erro-padrão de contagem de $0,7 \%$.

Enfim, de acordo com esses testes, referentes à solução cintiladora, concluiu-se que a temperatura das amostras, durante a contagem no cintilador (considerando-se o aparelho não refrigerado), não deve exceder a $22^{\circ} \mathrm{C}$, para não haver a separação de fases na emulsão Instage1-ägua do mar. Ainda, devem ser utilizadas alíquotas de $10 \mathrm{ml}$ dos filtrados, adicionadas a $10 \mathrm{ml}$ de Instage1. Os volumes menores que $2 \mathrm{ml}$ apresentam o inconveniente de, em amostras com pequena atividade, apresentarem contagens muito próximas às do $\mathrm{Bg}$. Além disso, o fato de serem multiplicados os resultados (em DPM) por fatores muito altos para a avaliação da MOD no volume total dos filtrados pode aumentar o erro das medidas.

\section{Preparo dos controles $(\mathrm{Bg})$}

Outro ponto crítico do método, estā relacionado com o controle, isto é, com o preparo de "brancos" para a ulterior correção das contagens espürias

("background" ou $\mathrm{Bg}$ ), devidas a outras causas que não a atividade biológica.

Vários autores têm criticado os altos valores de excreção encontrados em populações naturais e culturas. Esses valores, seriam decorrentes de contaminação nas ampolas de $\mathrm{NaH}^{14} \mathrm{CO}_{3}$ utilizadas e também do ${ }^{14} \mathrm{C}$ inorgânico não eliminado completamente dos filtrados pelo processo de borbulhamento (Morris etal., 1971; Thomas, 1971; Nalewajko \& Lean, 1972; Herbland \& Dandonneau, 1975; Steemann-Nielsen, 1975; Bul'Yon, 1976; Sharp, 1977; Wiebe \& Smith, 1977). Estes aspectos são analisados a seguir, separadamente.

\section{Contaminação das ampolas}

Em testes realizados com ampolas de vá-

Tabela II - Reprodutibilidade das contagens em solução cintiladora (Instagel) com diferentes volumes de uma solução glicerol ${ }^{14} \mathrm{C}(\tilde{0} 0,025$ $\mu \mathrm{C} / \mathrm{ml}$ ), em ägua do mar (Erro-padrão de contagem $=0,7 \%$ )

\begin{tabular}{|c|c|c|c|c|c|c|c|}
\hline \multicolumn{3}{|c|}{$\begin{array}{c}\text { Em cada frasco com } 10 \mathrm{mI} \\
\text { de Instagel }\end{array}$} & $I P M$ & $\begin{array}{l}\text { Eficiēncia de } \\
\text { contagem (z) }\end{array}$ & DPM & $D P M / m !$ & $\begin{array}{l}\text { Desvio da } \\
\text { média (z) }\end{array}$ \\
\hline Sol uçäo & $\begin{array}{l}\text { de } g 1 \text { icerol }{ }^{-14} \mathrm{c} \\
\text { (m1) }\end{array}$ & $\begin{array}{l}\text { Agua do mar } \\
(\text { (ml) }\end{array}$ & & & & & \\
\hline & 1 & 9 & 49888 & 81,6 & 61137 & 61137 & 1.0 \\
\hline & 2 & 8 & 98188 & 80,0 & 122735 & 61367 & 1,4 \\
\hline & 3 & 7 & 145854 & 80,0 & 182318 & 60773 & 0,4 \\
\hline & 4 & 6 & 192176 & 79.8 & 240822 & 60206 & 0,6 \\
\hline & 5 & 5 & 239196 & 79,6 & 300497 & 60098 & 0.7 \\
\hline & 6 & 4 & 288549 & 79,6 & 362499 & 60417 & 0,2 \\
\hline & 7 & 3 & 346280 & 81.4 & 425405 & 60772 & 0.4 \\
\hline & 8 & 2 & 401451 & 82,8 & 484844 & 60605 & 0,1 \\
\hline & 9 & 1 & 444372 & 82,0 & 541735 & 60193 & 0.6 \\
\hline & 10 & 0 & 485343 & 81,0 & 599188 & 59919 & 1.0 \\
\hline
\end{tabular}


rios lotes e procedếncias, realmente foi verificado que ocorre contaminação, não só por matéria orgânica dissolvida marcada, como também por material particulado que é retido pelos filtros. Vários pesquisadores também encontraram esse tipo de contaminação (Morris et al., 1971; Thomas, 1971; Williams et al., 1972).

Para verificar se as ampolas utilizadas apresentavam contaminação por material orgânico radioativo dissolvido, efetuou-se o seguinte teste: de cada 1ote de ampolas, retirou-se 10 ao acaso, das quais 5 foram irradiadas durante 72 horas por luz ultra-violeta (germicida) proveniente de 5 lâmpadas de $30 \mathrm{~W}$ cada, para fotooxidação da matēria orgânica. Após a irradiação, cada ampola foi adicionada a um frasco de incubação, contendo $200 \mathrm{ml}$ de água do mar filtrada e esterilizada. Em seguida, cada amostra foi tratada de acordo com a metodologia já descrita. As outras 5 ampolas foram utilizadas da mesma forma, porém, sem irradiação prévia. Para se verificar a contaminação por material particulado, após a filtração das amostras, os filtros foram lavados com $200 \mathrm{ml}$ de água destilada e expostos por 5 minutos a vapores de HCl. Suas margens não aproveitadas como superfície filtrante foram cortadas. A seguir, foram dissolvidos em solução de Bray.

Pela Tabela TII, verifica-se que a quantidade de ${ }^{14} \mathrm{C}$ residual é grande, mesmo após 30 minutos de borbulhamento. Ainda, esta atividade residual varia de ampola para ampola, dentro de um mesmo lote, tanto nos filtrados como nos filtros. Houve uma evidente queda dos seus valores nas ampolas irradiadas. Porém, a atividade continuou alta. Esses valores são provavelmente devidos à contaminação não eliminada pela irradiação de ultra-violeta em virtude da baixa potência da fonte, ou essa contaminação não è, em sua totalidade, fotooxidäve1. Poderiam também ser devidos à deficiente remoção do ${ }^{1{ }^{4}} \mathrm{C}$ inorgânico pelo método de borbulhamento. Como se viu na Tabela I, mesmo após 180 minutos de aeração, as atividades (em DPM) dos filtrados ainda foram relativamente a1tas, comparadas com o $\mathrm{Bg}$ do experimento (65 DPM).

A atividade residual nas ampolas irradiadas NEN (New England Nuclear) foi também maior que nas ampolas AD (Agency
Denmark). Isso indica ser, a mesma, devida, principalmente, à contaminação e não à deficiente remoção de ${ }^{1{ }^{4} \mathrm{C}}$ residual pelo borbulhamento, pois as ampolas $A D$ irradiadas apresentaram uma atividade residual muito próxima à do $\mathrm{Bg}$ (Tab. III).

\section{Tabela III - Atividade residual (DPM) devida à presença de ma- terial dissolvido e par- ticulado em ampolas de $\mathrm{NaH}^{14} \mathrm{CO}_{3}$ de vários lotes}

\begin{tabular}{|c|c|c|c|c|}
\hline \multirow{2}{*}{$\begin{array}{l}\text { Ampolas } \\
\text { lote }\end{array}$} & \multicolumn{2}{|c|}{ Näo irradiadas } & \multicolumn{2}{|c|}{ Irradiedas } \\
\hline & Filterado & Filtro & Filtrado & Filtro \\
\hline & 353 & 6220 & 187 & 3957 \\
\hline NEN & 352 & 7223 & 185 & 6734 \\
\hline $670-044$ & 349 & 5959 & 188 & 7484 \\
\hline \multirow[t]{3}{*}{$(20 u C i)$} & 298 & 7163 & 190 & 6523 \\
\hline & 375 & 7051 & 186 & 7587 \\
\hline & 259 & 3217 & 140 & 3620 \\
\hline NEN & 204 & 3636 & 142 & 1886 \\
\hline $670-053$ & 200 & 3318 & 158 & 4108 \\
\hline \multirow[t]{3}{*}{$(10 u C i)$} & 242 & 3886 & 155 & 3238 \\
\hline & - & 3624 & - & - \\
\hline & 208 & 1984 & 111 & 764 \\
\hline NEN & 195 & 1473 & 99 & 1241 \\
\hline $670-079$ & 180 & 1238 & 100 & 775 \\
\hline \multirow[t]{3}{*}{$(10 u c i)$} & 173 & 2551 & 113 & 1809 \\
\hline & 125 & 2247 & 99 & 814 \\
\hline & 122 & 4923 & 101 & 1753 \\
\hline NEN & 123 & 3985 & 100 & 3257 \\
\hline $670-099$ & 121 & 3893 & 98 & 3348 \\
\hline \multirow[t]{2}{*}{$(10 \mu C i)$} & 121 & 3970 & 103 & 3825 \\
\hline & 124 & 4204 & 97 & 3753 \\
\hline$A D$ & 71 & 106 & 68 & 105 \\
\hline 248 & 79 & 112 & 68 & 78 \\
\hline$(4 \mu C i)$ & 76 & 126 & 69 & 80 \\
\hline
\end{tabular}

NEN = New England Nuclear

$A D$ - Agency Denmark

$B g=$ (somente $\mathrm{H}_{2} \mathrm{O}$ do mar) $=65 \mathrm{DPM}$

Com base nos resultados desses testes, a preparação dos controles proposta aqui é a seguinte: as ampolas de $\mathrm{NaH}^{14} \mathrm{CO}_{3}$ a serem utilizadas, são adicionadas a um volume de amostra natural ou de cultura, suficiente para ser subdividido em $4 \mathrm{frascos}$ de incubação - 2 transparentes, 1 preto e 1 controle ao qual é adicionado formol a $2 \%$ e $\mathrm{HgCl}_{2}$ a $0,2 \%$. 0 controle é incubado durante o mesmo tempo e nas mesmas condições dos frascos experimentais. Este procedimento é imprescindível para se garantir a eliminação das causas de erro 
Bolm Inst. oceanogr., S Paulo, 31(1), 1982

acima discutidas. Ainda, com a inclusão de um controle, pode-se eliminar o erro devido à adsorção do $\mathrm{NaH}^{14} \mathrm{CO}_{3}$ ao material particulado nos demais frascos experimentais (transparentes e preto).

\section{Atividade minima detectāvel da MOD}

Os descontos do Bg das medidas de excreção devem ser feitos com relativa segurança, para se evitar que valores proximos ao mesmo (em DPM), devidos unicamente à flutuação estatística das contagens, sejam considerados como que oriundos da excreção. Para isso, é necessário um pequeno procedimento estatístico.

Algumas réplicas do controle (Bg) são contadas várias vezes. Após a observação de que não há indicação de variação significativa entre as diferentes réplicas, calcula-se o valor médio agrupado de todas as medidas pela expressão:

$\overline{\mathrm{Bg}}=\frac{1}{\mathrm{n}_{\mathrm{Bg}}} \cdot \sum_{i=1}^{\sum_{\mathrm{Bg}}^{\mathrm{B}}} \cdot \mathrm{Bg}_{\mathrm{i}}$,

onde " $i$ " indica a i-ésima medida (contagem) do $\mathrm{Bg}$.

o desvio-padrão indicativo da dispersão das medidas do Bg é calculado segundo a expressão:

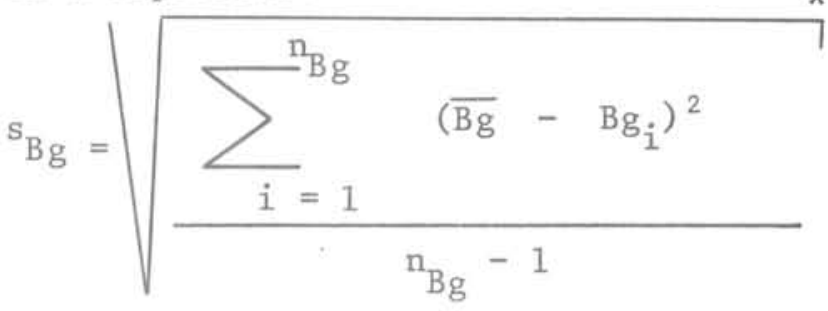

Para estabelecer a atividade mínima detectäve1 (AMD) da MOD, adotou-se a premissa de que a variância na determinação da mesma é praticamente igual àquela obtida para o Bg. A adoção dessa premissa não é propriamente verdadeira para os valores elevados da atividade

* Em casos em que a única componente da variância é a flutuação estatística da medida radioativa, o $\mathrm{s}_{\mathrm{Bg}}$ obedece a distribuiçấo de Poisson e $e^{\mathrm{Bg}}$ é estimado pela expressẫo $\mathrm{s}_{\mathrm{Bg}}=\sqrt{\overline{\mathrm{B}} \mathrm{g}}$. Entretanto, no presente caso, deve-se levar em conta outras componentes, tais como eventuais erros de subamostragens, e, portanto, preferiu-se utilizar a clássica fórmula para a estimativa do desviopadrão. da MOD. Entretanto, nesses casos, è evidente que o erro relativo na determinação da MOD é acentuadamente menor e, mediante a aplicação da expressão do teste $t$ de Student, constata-se que a premissa adotada é suficientemente criteriosa. A AMD da MOD é, então, estimada pela expressão:

$$
\mathrm{AMD}=\sqrt{2} \cdot \mathrm{t} \frac{\mathrm{s}_{\mathrm{Bg}}}{\mathrm{n}_{\mathrm{Bg}}}+\overline{\mathrm{Bg}} .
$$

0 valor de $t$ é lido em tabela apropriada (Snedecor \& Cochran, 1967). No presente caso, adotou-se o nível de $\mathrm{p}=$ 0,01 para inferências estatísticas e o número de graus de liberdade foi determinado pela expressão:

$$
\Phi=2 n_{B g}-2
$$

$\mathrm{Na}$ Tabela IV, que contém os resultados deste teste, $\Phi$ assumiu o valor de 6 e, consequentemente, $t=3,707$.

Para verificar a eficiência dos controles (Bg) na eliminação dos erros provenientes das contaminações das ampolas, ${ }^{14} \mathrm{C}$ residual nos filtrados, ou de qualquer outra fonte, realizou-se o seguinte teste:

Foram preparadas soluções de glicerol- ${ }^{14} \mathrm{C}$ em água do mar filtrada e esterilizada, com atividades teóricas de $1000,800,600,400,200,100,50,25$, 10 e $5 \mathrm{DPM} / 10 \mathrm{ml}$, a partir de uma solução-padrão, para simular diferentes concentrações de MOD marcada. Essas soluções foram divididas em duas partes iguais, sendo que a uma delas adicionouse $10 \mu \mathrm{Ci}$ de uma solução de $\mathrm{NaH}^{14} \mathrm{CO}_{3}$. Em seguida, as soluções foram tratadas como se fossem amostras de filtrados para a quantificação de excreção. Foram preparados controles para as medidas de $\mathrm{Bg}$ das soluções de glicerol- ${ }^{14} \mathrm{C}$, sem $\mathrm{NaH}^{14} \mathrm{CO}_{3}\left(\mathrm{Bg}_{1}\right)$ e com NaH ${ }^{14} \mathrm{CO}_{3}\left(\mathrm{Bg}_{2}\right)$.

Após o desconto do $\mathrm{Bg}$ encontrado nas soluções com $\mathrm{NaH}^{14} \mathrm{CO}_{3}$ (que simulam amostras reais), as contagens em DPM obtidas foram muito próximas daquelas encontradas nas soluções somente com glicerol- ${ }^{14} \mathrm{C}$ (Tab. IV). Pode-se observar, ainda, que o valor do $\mathrm{Bg}_{2}+8$ DPM (130 DPM) das soluções com $\mathrm{NaH}^{14} \mathrm{CO}_{3}$, permite detectar, no limite mínimo, apenas a "excreção" correspondente à atividade líquida de $25 \mathrm{DPM}$, uma vez que as solu- 
ções com 10 e 5 DPM, de glicerol $-{ }^{14} \mathrm{C}$, depois de adicionado $\circ \mathrm{NaH}^{14} \mathrm{CO}_{3}$, apresentaram 128 e 123 DPM, respectivamente; valores menores, portanto, que as 130 $\mathrm{DPM}$ do $\mathrm{Bg}_{2}$. Is so significa que, se fossem amostras reais, a MOD correspondente à excreção de uma população com uma atividade de 10 DPM em $10 \mathrm{ml}$ de alíquota dos filtrados, não teria significado estatístico.
MOD (em DPM) existente nos filtrados de células lavadas é insignificante, quando comparada à MOD presente antes das lavagens. 0 aumento verificado após 4 horas de incubação nas subamostras lavadas deve-se ao fato de que, nesse caso, foi efetuada apenas uma lavagem, ao invés de duas como nos demais horários.

0 teste é conclusivo quanto ao efeito da filtração, mas, se a MOD presente nos

Tabela IV - Utilização dos controles na discriminação entre a atividade devida à MOD (glicerol-14 $\mathrm{C}$ ) e ao ${ }^{14} \mathrm{C}$ residual das ampolas

\begin{tabular}{|c|c|c|c|c|}
\hline \multirow{2}{*}{$\begin{array}{l}\text { Agua d } \\
\text { Atividade } \\
\text { teörica }\end{array}$} & \multirow{2}{*}{\multicolumn{2}{|c|}{$\begin{array}{ll}\text { mar + Glicerol-1"C } & \text { (em } D P M) \\
\text { Atividade } & \text { Atividade obtida } \\
\text { obtida } & B g_{2}(=3,4)\end{array}$}} & \multicolumn{2}{|c|}{ Agua do mar + Glicerol-16 $\mathrm{C}+\mathrm{NaH}^{2}+\mathrm{CO}_{3}(\mathrm{em}$ OPM $)$} \\
\hline & & & $\begin{array}{c}\text { Atividade } \\
\text { obtida }\end{array}$ & $\begin{array}{c}\text { Atividade obtida } \\
\text { Bga }(=8,0)\end{array}$ \\
\hline 1000 & 1096 & 1028 & 1145 & 1023 \\
\hline 800 & 892 & 824 & 933 & 811 \\
\hline 600 & 680 & 612 & 728 & 606 \\
\hline 400 & 482 & 414 & 531 & 409 \\
\hline 200 & 327 & 259 & 376 & 254 \\
\hline 100 & 172 & 104 & 228 & 106 \\
\hline 50 & 121 & 53 & 173 & 51 \\
\hline 25 & 97 & 29 & 151 & 25 \\
\hline 10 & 75 & 7 & 128 & 6 \\
\hline 5 & 76 & 8 & 123 & 1 \\
\hline
\end{tabular}

$B g_{1}=68 \div 3,4 \mathrm{DPM}$ (intervalo de confiança ao nivel de $p=0,01$ )

$B g_{2}=122 \pm 8,0 \mathrm{DPM}$ (intervato de confiança ao nivel de $p=0,01$ )

Para se verificar o efeito da filtração na quantificação da excreção da MOD

(Arthur \& Rigler, 1967; Herbland, 1974; Sellner et al., 1976; Sharp, 1977), foi usada cultura de Phaeodactylum tricornutum em meio "f" $\operatorname{com} 20.10^{3}$ $\mathrm{cel} / \mathrm{ml}$. Adicionou-se $\mathrm{NaH}^{14} \mathrm{CO}_{3}(20 \mu \mathrm{Ci})$ e a incubação foi feita a $20^{\circ} \mathrm{C}$ e $\simeq$ 3.000 LUX.

A determinados intervalos de tempo (Fig. 5), foram retiradas amostras que foram subdivididas em duas partes. Uma delas, antes de ser filtrada, foi lavada duas vezes por centrifugação, para substituição do meio onde estavam crescendo as células, mais a possível MOD excretada por meio fresco de cultura. Dessa maneira, a atividade encontrada nessas subamostras seria devida ao efeito da filtração ou da excreção, durante o curto intervalo de tempo entre a ressuspensão em meio novo e a filtração. A outra subamostra sofreu tratamento normal, sem lavagem.

Os resultados mostram (Fig. 5) que a filtrados for proveniente de excreção natural ou de destruição de células ocorrida antes da filtração, pouco se poderá deduzir dos dados. 0 aumento acentuado, verificado em 200 horas de incubação, poderia ser causado por decomposição de células nesse período, mais que por excreção natural. No entanto, culturas de Phaeodactylum tricornutum nessas condições de cultivo, e com 8 dias de idade, apresentam-se ainda em fase exponencial de desenvolvimento; portanto, em boas condições fisiológicas. E pouco provável que a MOD encontrada seja devida à morte de células, considerandose a grande atividade encontrada no particulado. Deve, portanto, ser devida à MOD marcada acumulada.

Uma outra questão quanto ao efeito da filtração na quantificação da excreção de MOD é se, com o aumento da densidade celular sobre o filtro, a MOD, nos filtrados, aumenta como consequêencia de danos às células durante uma filtração mais demorada. Para se averiguar isso, um outro teste foi feito, utilizando-se 
Bolm Inst. oceanogr., S Paulo, 31(1), 1982

três espēcies diferentes: Phaeodactylum tricornutum, Skeletonema costatum (Baccilariophyta) e Tetraselmis sp (Chlorophyta).

Foram filtradas diferentes concentrações de células, obtidas por diluição de mesma cultura-mãe $\left(2.10^{6} \mathrm{cel} / \mathrm{m} 1\right)$.

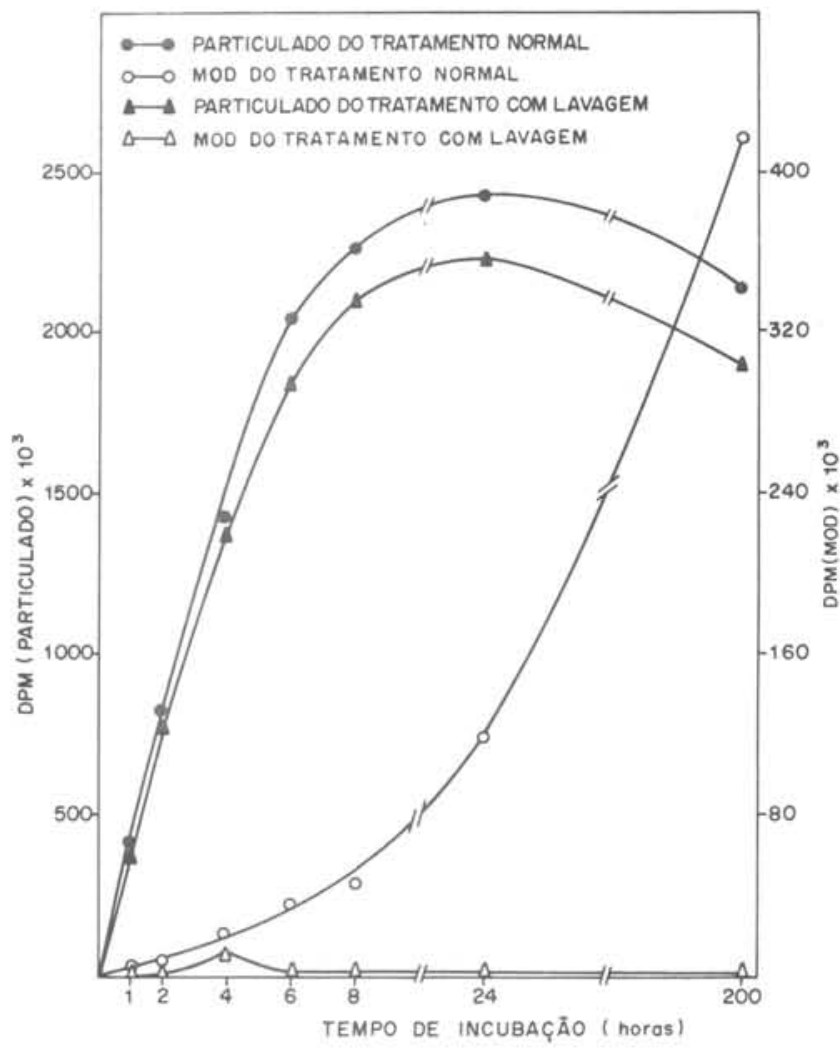

Fig. 5. Efeito da filtração na quantificação de MOD em culturas de Phaeodactylum tricornutum. Dados, em DPM, referentes à fração de carbono particulado e dissolvido de subamostras com tratamento normal e com lavagem das cēlulas por centrifugação.

Os resultados obtidos ( $\mathrm{Tab} . \mathrm{V}$ ) mostram que o aumento da concentração celular sobre o filtro não altera significativamente o valor da excreção. 0 que se observou, foi um aumento da MOD nos filtrados, em concentrações menores de células. Esse fenômeno ainda não foi bem esclarecido e os dados bibliográficos são, muitas vezes, contraditórios. Contudo, esse efeito é explicado por muitos autores, como sendo devido às grandes diluições: as células excretariam mais MOD para a manutenção de um equilíbrio intra/extra-celular de certos compostos (o ácido glicólico é o mais citado) (Tolbert \& Zill, 1956; Fogg et al., 1965; Ignatiades \& Fogg, 1973). 0 aumento da excreção poderia ser, ainda, devido a choques causados pela adição de meio fresco de cultura, sendo, portanto, uma causa não natural (Williams \& Yentsch, 1976). Grande parte das contradições sobre esse e outros aspectos da excreção, deve-se ao fato de se comparar dados obtidos com espécies pertencentes a ambientes diferentes (águas doce e salgada), crescendo em condições diversas, e até de classes taxonômicas distintas.

\section{Estocagem dos filtrados}

Em cruzeiros oceanogräficos e trabalhos no "campo", as medidas com equipamento de cintilação lîquida são praticamente impossiveis de serem feitas imediatamente após a coleta. De um modo geral, as amostras dos filtrados são estocadas e conservadas para serem preparadas para a contagem no laborátorio, algum tempo depois. Para se testar a validade da estocagem dos filtrados congelados e conservados durante longos períodos antes das contagens, foram realizados a1guns testes.

Durante o cruzeiro oceanográfico FINEP VII no N/Oc. "Prof. W. Besnard", do IOUSP, em janeiro de 1978, quando foram feitos experimentos de excreção, as alíquotas dos filtrados foram adicionadas, após o borbulhamento, diretamente aos frascos de cintilação, juntamente com o líquido cintilador. Os frascos foram tampados com batoques de plástico inerte, além da tampa rosqueáve1, para evitar evaporação e, em seguida, congelados. Paralelamente, subamostras desses filtrados foram fixadas com $\mathrm{HgCl}_{2}$ a $0,2 \%$ e congeladas para medidas posteriores em diferentes intervalos de tempo.

Foram efetuadas medidas, não só das amostras conservadas, como também daquelas colocadas nos frascos de cintilação preparados a bordo, entre 30 e 77 dias após a coleta. As primeiras contagens não foram efetuadas imediatamente, mas somente depois de aproximadamente 10 dias, duração de cada uma das etapas do cruzeiro.

Para o teste, foram escolhidas amostras de três experimentos com baixa, média e alta atividades (estações 3501, 3391 e 3444, respectivamente) (Tab. VI). Como se pode verificar, as variações 
Tabela V - Fixação total de ${ }^{14} \mathrm{C}$ (em DPM) e da fração dissolvida em diferentes concentrações celulares obtidas por diluição das culturas-mãe

\begin{tabular}{|c|c|c|c|c|}
\hline Espēcie & Fixaçāo total & Dissolvida & \% Dissolvida & $\begin{array}{c}\text { Concentraçäo } \\
\text { relativa } \\
\text { cel. } 1 . \mathrm{ml}\end{array}$ \\
\hline & 499.048 & 5456 & 1,1 & 100 \\
\hline & 498.378 & 5288 & 1,1 & 75 \\
\hline Phaeodactylum & 329.993 & 3496 & 1,1 & 50 \\
\hline \multirow[t]{5}{*}{ tricornutum } & 160.443 & 2160 & 1,3 & 25 \\
\hline & 61.900 & 1200 & 1,9 & 10 \\
\hline & 6.670 & 136 & 2,0 & 1 \\
\hline & 660.625 & 3380 & 0,5 & 100 \\
\hline & 501.896 & 3680 & 0,7 & 75 \\
\hline Tetraselmis & 220.470 & 1955 & 0,9 & 50 \\
\hline \multirow[t]{5}{*}{ sp } & 159.714 & 1480 & 0,9 & 25 \\
\hline & 61.607 & 1045 & 1,7 & 10 \\
\hline & 9.666 & 995 & 10,3 & 1 \\
\hline & 1.211 .361 & 14450 & 1,2 & 100 \\
\hline & 1.000 .676 & 11747 & 1,2 & 75 \\
\hline Skeletonema & 773.341 & 8704 & 1,1 & 50 \\
\hline \multirow[t]{3}{*}{ costatum } & 406.928 & 5143 & 1,2 & 25 \\
\hline & 174.922 & 3324 & 1,9 & 10 \\
\hline & 35.539 & 1556 & 4,4 & 1 \\
\hline
\end{tabular}

Tabela VI - Variação da atividade (em DPM) com o tempo de estocagem em líquido cintilador e das subamostras conservadas (Erro-padrão de contagem $=$ $2 \%$ )

\begin{tabular}{|c|c|c|c|c|c|c|c|c|c|c|c|c|}
\hline \multirow{2}{*}{$\begin{array}{c}\text { Estaçäo e } \\
\text { Data de coleta }\end{array}$} & \multirow{2}{*}{ Frasco } & \multicolumn{2}{|c|}{ A } & \multicolumn{3}{|c|}{ B } & \multicolumn{3}{|c|}{ c } & \multicolumn{3}{|c|}{ D } \\
\hline & & DPM & E & DPM & E & $F$ & DPM & E & $F$ & DPM & $\varepsilon$ & $F$ \\
\hline \multirow{3}{*}{$\begin{array}{c}3391 \\
(13 / 01 / 78)\end{array}$} & $T_{1}$ & 311 & & 311 & & 0,0 & 308 & & 1,0 & 304 & & 2,3 \\
\hline & $T_{2}$ & 307 & 7 & 305 & 77 & 0,7 & 302 & 48 & 1,0 & 299 & 77 & 2.6 \\
\hline & P & 128 & , & 128 & & 0,0 & 136 & & 6.0 & 136 & & 6,0 \\
\hline \multirow{3}{*}{$\begin{array}{c}3444 \\
(23 / 01 / 78)\end{array}$} & $T_{1}$ & 1390 & & 1387 & & 0,2 & 1386 & & 0.3 & 1386 & & 0,3 \\
\hline & $T_{2}$ & 1320 & 10 & 1323 & 68 & 0,2 & 1319 & 38 & 0,1 & 1299 & 68 & 1,6 \\
\hline & $P$ & 252 & & 258 & & 2,3 & 248 & & 1,6 & 228 & & 9,5 \\
\hline \multirow{3}{*}{$\begin{array}{c}3501 \\
(30 / 01 / 78)\end{array}$} & $T_{1}$ & 175 & & 177 & & 1,1 & 175 & & 0,0 & 159 & & 9,1 \\
\hline & $T_{2}$ & 178 & 3 & 170 & 61 & 4,5 & 168 & 31 & 5,6 & 160 & 61 & 5.6 \\
\hline & $P$ & 138 & & 140 & & 1,4 & 137 & & 0,0 & 132 & & 4,3 \\
\hline
\end{tabular}

$A=$ Primeira contagem das amostras em liquido cintilador, preparadas a bordo $(20 / 01 / 78$ e $02 / 02 / 78)$

$B=$ Recontagem de $A$ em $01 / 04 / 78$

$\mathrm{CeD}=$ Contagem de subamostras dos filtrados em 02/03/78 (C) e em 01/04/78 (D). conservadas com HgCl2 a $0,2 \%$ e mantidas congeladas

$E=$ Tempo, em dias, em que as amostras ficaram congeladas desde a coleta

$F=$ Variação (\%) das contagens em relação a A 
Bolm Inst. oceanogr., S Paulo, 31(1), 1982

nas contagens após 77 dias nos frascos de cintilação preparados a bordo, são despreziveis e estão dentro do erropadrão de contagem ( $2 \%$ ), com exceção daquelas referentes ao frasco $\mathrm{T}_{2}$ (transparente) da estação 3501, após 61 dias de coleta. As medidas das subamostras mantidas congeladas por até 48 dias também não variaram significativamente, quando comparadas com as primeiras contagens dos frascos de cintilação preparados a bordo. As maiores variações, verificadas em $P$ (frasco preto da estação 3391) e $\mathrm{T}_{2}$ da estação 3501 , provavelmente foram devidas a erros nas subamostragens. Entretanto, verificou-se um acentuado decréscimo nas contagens das subamostras após 61 dias de estocagem, evidenciando uma perda de atividade.

Para se comparar medidas de amostras nos frascos de cintilação feitas imediatamente após as coletas dos filtrados ( 3 horas, tempo necessário para a eliminação do efeito da quimioluminiscência), com medidas feitas posteriormente (30 a 90 dias) dos mesmos frascos guardados congelados, foram utilizados filtrados de uma cultura de Phaeodactylum tricornutum crescendo em fase exponencial. Os resultados são apresentados na Tabela VII. Observa-se que, no espaço entre 3 horas e 90 dias de preparação das amostras nos frascos de cintilação, a atividade permaneceu a mesma.

Tabela VII - Variação da atividade (DPM) com o tempo de es tocagem das amostras de filtrados de Phaeodactylum tricornutum em frascos de cintilação; os nümeros entre parênteses indicam a variação, em \%, em relação à primeira contagem (Erropadrão de contagem $=2 \%$ )

\begin{tabular}{l|cccc}
\hline \multirow{2}{*}{$\begin{array}{c}\text { Frasco } \\
\text { (incubaçäo) }\end{array}$} & \multicolumn{4}{|c}{ Tempo após a colecta dos fitcrados } \\
\cline { 2 - 5 } & 3 horas & 30 dias & 60 dias & 90 dias \\
\hline \multirow{2}{*}{ Transparente } & 4705 & 4710 & 4687 & 4632 \\
& & $(0,1)$ & $(0,4)$ & $(1,6)$ \\
& 4734 & 4722 & 4703 & 4641 \\
Transparente & & $(0,3)$ & $(0.7)$ & $(2,0)$ \\
& & 193 & 190 & 188 \\
Preto & 192 & $(0.5)$ & $(1,1)$ & $(2,1)$ \\
\hline
\end{tabular}

Pelos experimentos acima, é possîvel concluir que, no caso de trabalhos de campo, ou quando não houver possibilidade de contagem imediata das amostras, estas devem ser guardadas preparadas nos frascos de cintilação e congelados. Este procedimento é preferivel ao simples congelamento dos filtrados e posterior preparação para contagem em equipamento de cintilação líquida.

\section{Considerações finais}

A técnica de medida da MOD por cintilação líquida é de fácil execução e grande sensibilidade, permitindo precisão nos resultados.

0 método permite detectar se ocorreu liberação de MOD pelo fitoplâncton; is to é, se a radioatividade medida nos filtrados é proveniente da excreção, e não devida aos processos metodológicos. Entretanto, não discrimina a MOD excretada por um processo natural daquela proveniente da morte e decomposição de células durante o período do experimento. Contudo, pode-se argumentar que, se em populações naturais, durante o período de exposição à $1 \mathrm{uz}$ em presença de $\mathrm{NaH}^{1{ }^{4}} \mathrm{CO}_{3}$, existem células que morrerão devido ao seu estado fisiológico alterado, este mesmo estado não permitiria a essas células uma fotossíntese tal que, durante o tempo relativamente curto de incubação, contribuísse significativamente para o aumento da MOD nos filtrados. Também, deve-se considerar o tempo necessário para a desintegração de uma célula que tenha assimilado carbono e uma taxa normal e que, por qualquer motivo, venha a morrer durante a incubação.

$\mathrm{Se}$, em um experimento, o número de células em estado fisiológico "precário" ou senescentes for elevado, elas assimilarão $\circ{ }^{14} \mathrm{C}$ e o liberarão em taxas elevadas. Ecologicamente, porém, este fato é irrelevante, uma vez que na natureza não existem somente células saudáveis.

Finalmente, é importante ressaltarse que, se o método elimina as principais fontes de erro que superestimam a excreção, não elimina outras que podem subestimá-1a. Entre estas, pode-se citar a ação bacteriana sobre os compostos excretados, que os transforma em matérĩa orgânica particulada, a volatilização de certos compostos de baixo peso 
molecular durante o borbulhamento

(Anderson \& Zeutsche1, 1970; Eppley \& Sloan, 1965; Hellebust, 1974) e a transformação da MOD em colóides devido ao borbulhamento, o que acarretaria medidas erräticas nas réplicas dos filtrados (Wangersky, 1965).

\section{Agradecimentos}

Os autores agradecem ao MSc Carlos Henrique Mesquita do Instituto de Pesquisas Energéticas e Nucleares (IPEN) pelas valiosas críticas e sugestões recebidas.

\section{Referências bibliogräficas}

ANDERSON, G. C. \& ZEUTSCHEL, R. P. 1970. Release of dissolved organic matter by marine phytoplankton in coastal and off-shore areas of the Northeast Pacific Ocean. Limnol. Oceanogr., 15:402-407.

ARMSTRONG, F. A. J.; WILLIAMS, P. M. \& STRICKLAND, J. D. H. 1966. Photooxidation of organic matter in sea water by ultra-violet radiation, analytical and other applications. Nature, Lond., 211:481-483.

ARTHUR, C. R. \& RIGLER, F. H. 1967. A possible source of error in the ${ }^{14} \mathrm{C}$ method of measuring primary productivity. Limnol. Oceanogr., 12:121-124.

BERMAN, R. \& HOLM-HANSEN, 0. 1974. Release of photoassimilated carbon as dissolved organic matter by marine phytoplankton. Mar. Biol., $28: 305-310$.

BRANDSOME Jr., E. D. 1970. The current status of liquid scintillation counting. New York, Grune \& Stratton.

BRAY, G. A. 1960. A simple efficient liquid scintillator for counting aqueous solutions in liquid scintillation. Anal. Biochem., $1: 279-285$.

BUL'YON, V. V. 1976. Phytoplankton production measurement using $\mathrm{C}^{14}$ with allowance for losses of labeled organic matter during filtering. Oceanology, Acad. Sci. URSS., $15(3): 347-350$.
EHRHARDT, M. 1977. Organic substances in seawater. Mar. Chem., 5:307-316.

EPPLEY, R. W. \& SLOAN, P. R. 1965. Carbon balance experiments with marine phytoplankton. J. Fish. Res. Bd Can., 22:1083-1097.

FOGG, G. E. 1958. Extracellular products of phytoplankton and the estimation of primary production. Rapp. P.-v. Réun. Cons. perm. int. Exp1. Mer, 144:56-60.

; NALEWAJKO, C. \& WATT, W. D. 1965. Extracellular products of phytoplankton photosynthesis. Proc. R. Soc., B., 162:517-534.

GUILLARD, R. R. L. \& RYTHER, J. H. 1962. Studies of marine planktonic diatoms. I. Cyclotella nana Ustedt, and Detonula confervacea (Cleve) Gran. Can. J. Microbiol., 8:229-239.

HELLEBUST, J. A. 1974. Extracellular products. In: Stewart, W. D. P., ed. - Algae physiology and biochemistry. Oxford, Blackwe11, p. 838-863.

HERBLAND, A. 1974. Influence de 1a dépression de filtration sur 1 a mesure simultanée de 1 'assimilation et de 1 'excrétion organique du phytoplancton. CUEA Newsletter, $3(4): 16-22$.

\& DANDONEAU, Y. 1975.

Excrétion organique du phytoplancton et activité bactérienne hétérotrophe dans le Dôme de Guinée (Océan Atlantique Tropical Est). Doc. scient. Centre Rech. océanogr. (Abidjan), 6(2):1-18.

IGNATIADES, L. \& FOGG, G. E. 1973. Studies on the factors affecting the release of organic matter by Skeletonema costatum (Greville) Cleve in culture. J. mar. biol. Ass. U.K., 53:937-956.

JøRGENSEN, C. B. 1976. August Pütter, August Krogh, and modern ideas on the use of dissolved organic matter in aquatic environments. Biol. Rev., $51: 291-328$, 
KROGH, A. 1934a. Conditions of life in the ocean. Ecol. Monogr., 4:421429.

1934b. Conditions of 1 ife at great depths in the ocean. Ecol. Monogr., 4:430-439.

\& KEYS, A. 1934. Methods for the determination of dissolved organic carbon and nitrogen in sea water. Biol. Bull. mar. Biol. Lab., Woods Hole, $67(1): 132-144$.

; LANGE, E. \& SMITH, W. 1930. On the organic matter given off by algae. Biochem. J., 24:1666-1671.

MORRIS, I.; YENTSCH, C. M. \& YENTSCH, C. S. 1971. Relationships between light carbon dioxide fixation and dark carbon dioxide fixation by marine algae. Limno1. Oceanogr., $16: 854-858$.

MYERS, J. \& JOHNSTON, J. A. 1949. Carbon and nitrogen balance of Chlorella during growth. Plant. Physiol., 24:111-119.

NALEWAJKO, C. \& LEAN, D. R. S. 1972. Retention of dissolved compounds by membrane filters as error in the ${ }^{14} \mathrm{C}$ method of primary production measurement. J. Phycol., 8:37-43.

PATTERSON, M. S. \& GREENE, R. C. 1965. Measurement of low energy betaemitters in aqueous solution by liquid scintillation counting of emulsions. Ana1. Chem., 37:854-857.

SELLNER, K. G.; ZINGMARK, R. G. \& MILLER, T. G. 1976. Interpretations

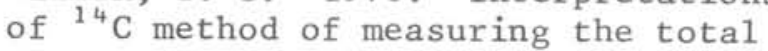
annual production of phytoplankton in the South Caroline estuary. Bot. mar., 19:119-125.

SHARP, J. H. 1977. Excretion of organic matter by marine phytoplankton: do healthy cells do it? Limno1. Oceanogr., 22:381-399.

SMITH Jr., W. O. 1975. The optimal procedure for the measurement of phytoplankton excretion. Mar. Sci. Commun., 1(6):395-405.
SNEDECOR, G. W. \& COCHRAN, W. G. 1967. Statistical methods. 6th ed. Ames, Iowa, Iowa State University Press.

STEEMANN-NIELSEN, E. 1952. The use of radioactive carbon $\left({ }^{14} \mathrm{C}\right)$ for measuring organic production in the sea. J. Cons. perm. int. Explor. Mer, 18:117-140.

1975. Marine
photosynthesis. Amsterdam, E1sevier,
141p.

THOMAS, J. P. 1971. Release of dissolved organic matter from natural populations of marine phytoplankton. Mar. Bio1., 11:311-323.

TOLBERT, N. E. \& ZILL, L. P. 1956. Excretion of glicolic acid by some algae during photosynthesis. J. Biol. Chem., 222:895-906.

TURNER, J. C. 1968. Triton $x-100$ scintillant for carbon labelled materials. Int. J. App1. Radiat. Isotopes, 19:557-563.

WANG, C. H. \& WILLIS, D. L. 1965. Radiotracer methodology in biological science. Englewood Eliffs, PrenticeHal1, 382p.

WANGERSKY, P. J. 1965. The organic chemistry of sea water. Am. Scient., $53: 358-374$.

WIEBE, W. J. \& SMITH, D. F. 1977. ${ }^{14} \mathrm{C}-1$ abe11ing of compounds excreted by phytoplankton for employment as a realistic tracer in secondary productivity measurement. Microbiol. Ecol., 4:1-8.

WILLIAMS, P. J. de B. \& YENTSCH, C. S. 1976. An examination of photosynthesis production, excretion of photosynthetic products and heterotrophic utilization of dissolved organic compounds with 
reference to results from coastal

sub-tropical sea. Mar. Biol.,

$35: 31-40$.

WILLIAMS, P. J. de B.; BERMAN, T. \&

HOLM-HANSEN, O. 1972. Potential

sowires of error in the measurement

of low rates of planktonic

photosynthesis and excretion.

Nature, New Biol., 236:91-93.

(Manuscrito, recebido em $23 / f e v . / 1981$;

acei to em 25/fev./1982) 